\title{
Sublethal dose of irradiation enhances invasion of malignant glioma cells through p53-MMP 2 pathway in U87MG mouse brain tumor model
}

Jian Pei ${ }^{1}$, In-Ho Park ${ }^{1}$, Hyang-Hwa Ryu ${ }^{2}$, Song-Yuan Li ${ }^{2}$, Chun-Hao Li' ${ }^{2}$ Sa-Hoe Lim,2, Min Wen², Woo-Youl Jang ${ }^{1}$ and Shin Jung ${ }^{1,2^{*}}$

\begin{abstract}
Background: Glioblastoma is a highly lethal neoplasm that frequently recurs locally after radiotherapy, and most of these recurrences originate from near the irradiated target field. In the present study, we identified the effects of radiation on glioma invasion and p53, TIMP-2, and MMP-2 expression through in vitro and in vivo experiments.

Methods: The U87MG (wt p53) and U251 (mt p53) human malignant glioma cell lines were prepared, and the U2OS (wt 53) and Saos2 (del p53) osteosarcoma cell lines were used as p53 positive and negative controls. The four cell lines and p53 knock-downed U87MG cells received radiation (2-6 Gy) and were analyzed for expression of p53 and TIMP-2 by Western blot, and MMP-2 activity was detected by zymography. In addition, the effects of irradiation on directional invasion of malignant glioma were evaluated by implanting nude mice with bioluminescent u87-Fluc in vivo followed by MMP-2, p53, and TIMP-2 immunohisto-chemistry and in situ zymography.

Results: MMP-2 activity and p53 expression increased in proportional to the radiation dose in cell lines with wt p53, but not in the cell lines with del or mt p53. TIMP-2 expression did not increase in U87MG cells. MMP-2 activity decreased in p53 knock-downed U87MG cells but increased in the control group. Furthermore, radiation enhanced MMP-2 activity and increased tumor margin invasiveness in vivo. Tumor cells invaded by radiation overexpressed MMP-2 and p53 and revealed high gelatinolytic activity compared with those of non-radiated tumor cells.

Conclusion: Radiation-induced upregulation of p53 modulated MMP-2 activity, and the imbalance between MMP-2 and TIMP-2 may have an important role in glioblastoma invasion by degrading the extracellular matrix. Bioluminescent "U87-Fluc"was useful for observing tumor formation without sacrifice after implanting tumor cells in the mouse brain. These findings suggest that the radiotherapy involved field for malignant glioma needs to be reconsidered, and that future trials should investigate concurrent pharmacologic therapies that inhibit invasion associated with radiotherapy.
\end{abstract}

Keywords: Radiation, Glioblastoma, p53, MMP-2, TIMP-2, Sublethal dose

\footnotetext{
* Correspondence: sjung@jnu.ac.kr

'Department of Neurosurgery, Chonnam National University Hwasun Hospital and Mediacal School, 322 Seoyang-ro, Hwasun-eup, Hwasun-gun, Jeonnam 519-763, Republic of Korea

${ }^{2}$ Brain Tumor Research Laboratory, and Chonnam National University

Research Institute of Medical Sciences, Chonnam National University Hwasun

Hospital and Medical School, 322 Seoyang-ro, Hwasun-eup, Hwasun-gun,

Jeonnam 519-763, Republic of Korea
} 


\section{Background}

Glioblastoma (GBM) is the most malignant of primary adult brain tumors and is characterized by a highly localized invasive cell population, abundantly proliferative cells, neoangiogenesis, and necrosis. The current treatment standard involves a multimodal approach of neurosurgery, fractionated radiotherapy (RT), and chemotherapy [1]. However, despite continuous improvements in GBM treatment, median survival time does not exceed 15 months [2].

$\mathrm{RT}$ is the main treatment for brain tumors. The RT target volume consists of the tumor volume with a $2-3 \mathrm{~cm}$ margin of surrounding tissue, which is considered to be at risk for microscopic tumor invasion. Traditional targetvolume RT has raised a question of whether RT could be helpful for killing tumor cells that have infiltrated the surrounding normal brain. In fact, whole brain RT has not decreased the risk of recurrence and has seriously increased the risk of neuropsychological deficits, e.g., dementia [3]. Furthermore up to $90 \%$ of all gliomas relapse in close proximity to the resection cavity or the postoperative RT target volume $[4,5]$. In other words, it may be important to understand the biological characteristics of invading cells into the surrounding brain tissue. Invasive tumor cells have a G0 phase, are resistant to RT and chemotherapy, and eventually regrow. G0/G1 phase cells migrate more rapidly and further than cancer cells in the S/G2/M phases. Cancer cells cease migrating when they enter the $S / G 2 / M$ phases and restart migration after cell division when they re-enter the G0/G1 phase [6]. Radiation to surrounding brain tissue augments secretion of several mediators, including stromal cell-derived factor$1 \alpha$, vascular endothelial growth factor (VEGF) and matrix metalloproteinases (MMPs), which play a vital role in tumor invasion and metastasis [7].

MMPs are a family of zinc-dependent proteolytic endopeptidases that break down the structural barriers to migration and invasion by dissolving and destroying the matrix proteins of surrounding normal brain tissue $[8,9]$. In 2001, Wild-Bode et al. reported that sublethal doses of radiation promote glioma invasion [10]. Another study suggested that this may be mediated by MMPs, such as MMP-2, as irradiation increases MMP-2 levels [11]. In particular, MMP-2 and 9 are highly expressed in GBM compared with that in normal brain tissue [1]. Ionizing radiation enhances MMP-2 production in a variety of human cancers, and activation of wild-type p 53 by radiation and the resulting increase in MMP-2 expression suggest that radiation may promote invasion-related gene expression [12-15].

p53, a 53-kDa nuclear protein, is one of the most extensively studied molecules in cancer research and molecular biology. p53 has many anticancer functions and plays critical roles in DNA repair, apoptosis, and inhibiting angiogenesis [16]. Wild-type p53 is required to activate apoptosis in response to DNA damage [17] and, thus, may be a critical determinant of the effectiveness of ionizing radiation and chemotherapeutic agents. According to a 1995 report, patients with cancer harboring a p53 mutation often have poorer prognoses than those with tumors that harbor wild-type p53 [18]. Thus, the genetic and functional status of the p53 gene is an important factor guiding therapeutic strategies in patients with cancer [19]. In 1997, after a computer search for other potential p53 target genes, Bian et al. determined that wild-type p53 binds the promoter of the gene encoding human type IV collagenase (also called gelatinase A or MMP-2), a naturally occurring enzyme subject to inhibition by TIMP-2, among other known p53 target genes $[14,20]$.

In this study, we investigated the effects of radiation on malignant glioma invasion and p53, TIMP-2, and MMP-2 expression through in vitro and in vivo experiments. As focal brain irradiation remains the standard of care for managing malignant glioma, understanding the effects of RT on invasion of malignant glioma may impact RT strategy. We hope that our findings will help understanding the changes occurring in surrounding normal brain tissues within the postoperative RT field and provide a basis for explaining local relapse during or a few months after RT.

\section{Materials and methods}

\section{Cell culture conditions}

The human malignant glioma cell lines U87MG (wt p53) and U251 (mt p53) and the human osteosarcoma cell lines U2OS (wt p53) and SAOS (del p53) were acquired from the American Type Culture Collection (Manassas, VA, USA). The U87-Fluc cell line, which is transfected with a lentiviral vector containing the firefly luciferase (Fluc) gene, was a gift from Professor Min (Hwasun Chonnam National University Hospital, Korea). The cell lines were routinely maintained in high-glucose DMEM (Gibco BRL, Gaithersburg, MD, USA) supplemented with $10 \%$ fetal bovine serum (Gibco) and maintained at $37^{\circ} \mathrm{C}$ containing $5 \% \mathrm{CO}_{2} / 95 \%$ air.

\section{siRNA oligonucleotide transfection}

A siRNA oligonucleotide was used for p53 knockdown. The synthesized p53 siRNA (5'-CACUACAACUACAU GUGUA-3') and scrambled RNA (negative control) were purchased from Bioneer (Daejeon Korea). Approximately $2 \times 10^{5}$ u87MG cells were seeded on a plate and transfected with the siRNA oligonucleotide using Lipofectamine $^{\mathrm{Tm}}$ RNAiMAX (Invitrogen, Carlsbad, CA, USA), according to the manufacturer's instructions. p53 knockdown was confirmed by Western blotting. 


\section{Radiation}

The cell lines were grown in $60 \mathrm{~mm}$ culture dishes until 80-90\% confluent and then the media were replaced with serum free media to synchronize the cell cycle. The cells were exposed to radiation from a Gammacell 1000 unit ( ${ }^{137} \mathrm{Cs}$; Nordion, Kanata, ONT, Canada) at 2, 4, and 6 Gy. The irradiated cells were further cultured for $24 \mathrm{~h}$ and harvested.

\section{Preparation of total protein and conditioned medium}

The cells were lysed with a protein extraction buffer [50 mM Tris ( $\mathrm{pH} 8.0$ ), $5 \mathrm{mM}$ EDTA, $150 \mathrm{mM}$ sodium chloride, $0.5 \%$ deoxycholic acid, $0.1 \%$ sodium dodecyl sulfate (SDS), $1 \%$ NP-40, 1 mM phenylmethylsulfonyl fluoride, and $1 \mathrm{mg} / \mathrm{ml}$ protease inhibitor cocktail] to prepare total protein. The cells were grown in $60 \mathrm{~mm}$ culture dishes until subconfluent, and the media were replaced with serum-free medium to prepare the conditioned medium. After radiation exposure, the cells were incubated at $37^{\circ} \mathrm{C}$ for $24 \mathrm{~h}$. The conditioned media were clarified by centrifugation, and protein concentrations were determined using a protein assay kit (Bio-Rad, Hercules, CA, USA).

\section{Western blot}

Whole cell lysates $(20 \mu \mathrm{g})$ were separated by $8-10 \%$ SDS-polyacrylamide gel electrophoresis and transferred to PVDF membranes (Pall Corp., Port Washington, NY, USA). The membranes were incubated for $2 \mathrm{~h}$ at room temperature with $5 \%$ non-fat dry milk, probed overnight at $4{ }^{\circ} \mathrm{C}$ with actin, p53, TIMP-2, and MMP-2 antibodies (Santa Cruz Biotechnology, Santa Cruz, CA, USA) and incubated with a horseradish peroxidaselabeled goat anti-rabbit IgG (Jackson Immunoresearch Laboratory, West Grove, PA, USA). Bound secondary antibody was detected by enhanced chemiluminescence (Amersham Biosciences, Bucks, UK), and protein levels were determined by autoradiography using a LAS-4000 instrument (Fuji, Tokyo, Japan).

\section{Gelatin zymography}

Twenty $\mu \mathrm{g}$ of proteins in conditioned media were mixed with sample buffer (50 mM Tris- $\mathrm{HCl}, 2 \%$ SDS, $0.1 \%$ bromophenol blue, and $10 \%$ glycerol) before electrophoresis. Aliquots were electrophoresed on $8 \%$ SDSpolyacrylamide gels containing $1 \mathrm{mg} / \mathrm{ml}$ type A gelatin (Sigma-Aldrich, St. Louis, MO, USA). Each gel was washed three times for $30 \mathrm{~min}$ in $2.5 \%$ Triton X-100 and then incubated for $20 \mathrm{~h}$ at $37{ }^{\circ} \mathrm{C}$ in incubation buffer [50 mM Tris- $\mathrm{HCl}$ (pH 7.5), $10 \mathrm{mM} \mathrm{CaCl}_{2}$, and $200 \mathrm{mM}$ $\mathrm{NaCl}]$. The gels were stained with Coomassie Brilliant Blue R-250 (0.2 \% Coomassie Brilliant Blue R-250, 20 \% methanol, $10 \%$ acetic acid in water) and then destained in $20 \%$ methanol and $10 \%$ acetic acid in water.

\section{In vivo studies}

Five- to six-week old male BALB/c athymic nu-/nu mice (body weight, 20-30 g) were purchased from the Orient Co. (Seongnam, Korea). They were housed in groups of three or four under standard conditions at a temperature of $22{ }^{\circ} \mathrm{C}$ and a 12-h light/12-h dark cycle. The mice had free access to standard food pellets and tap water. The mice were anesthetized with isoflurane (2\%), and a mixture of ketamine $(200 \mathrm{mg} / \mathrm{kg}$ ) and xylazine $(10 \mathrm{mg} / \mathrm{kg})$ for irradiation. Radiation treatment was carried out 14-21 days after tumor implantation. Tumor growth was monitored twice weekly by optical bioluminescence imaging, and signals were observed in all animals 7 days after tumor implantation. All animal care, experiments, and euthanasia were performed in accordance with protocols approved by the Chonnam National University Animal Research Committee (Gwangju, Korea).

\section{Bioluminescence imaging}

Anesthetized mice were placed in the light-tight chamber of the IVIS 100 imaging system (Caliper, Newton, MA, USA), equipped with a cooled charge-coupled device camera to obtain the tumor bioluminescence images. Photons emitted from the luciferase-expressing tumor were collected and integrated over 1-min periods. Pseudocolor images indicating photon counts were overlaid on photographs of the mice using Living Image software v. 2.25 (Caliper). A region of interest was selected manually based on signal intensity. The area of the region of interest was kept constant, and intensity was recorded as maximum radiance within each region of interest.

\section{Radiotherapy}

RT was commenced 2 weeks after tumor implantation. A 6-MV X-ray was used via a linear accelerator (CLINAC 21EX; Varian, Palo Alto, CA, USA). The source to skin distance was $97.5 \mathrm{~cm}$ with a field size of $5 \times 5 \mathrm{~cm}^{2}$ and a dose rate of $3 \mathrm{~Gy} / \mathrm{min}$. Water equivalent boluses of $1 \mathrm{~cm}$ thickness were placed under and above the tumor-bearing mouse brain to establish dose homogeneity. Additional file 1: Figure S1 illustrates the process to assess the radiation effect on brain tumors in vivo. After implanting the U87-Fluc cells in the brain, tumor bioluminescence of mouse brain cells bearing U87-Fluc was observed continuously from day 10 to 28 using the IVIS 100 imaging system. Radiation exposure was either a single 6 Gy dose or 2 Gy fractions five times for 7 days on day 14 after U87-Fluc implantation. The mice brain tissue was stained with hematoxylin and eosin (H\&E) or immunohistochemical staining after killing the mice on day 30. 


\section{Histopathology}

All mice were anesthetized and perfused transcardially with 4 \% ZBF-containing $36.7 \mathrm{mM} \mathrm{ZnCl}$, $27.3 \mathrm{mM}$ $\mathrm{ZnAc}_{2} \cdot 2 \mathrm{H}_{2} \mathrm{O}$, and $0.63 \mathrm{mM} \mathrm{CaAc} 2$ in $0.1 \mathrm{M}$ Tris, $\mathrm{pH}$ 7.4. The brain tumor was removed, fixed in the same solution for $36-38 \mathrm{~h}$ at room temperature and then dehydrated for paraffin embedding. The tumors were blocked in cross-section and processed for paraffin embedding.

Four $\mu \mathrm{m}$-thick consecutive sections were cut from the recipient blocks and placed on poly-L-lysine-coated slides for immunohistochemistry. Representative sections were stained with H\&E. Heat-induced epitope retrieval was carried out for $10 \mathrm{~min}$ at $120{ }^{\circ} \mathrm{C}$ in a pressure cooker in 10 mM citrate buffer, pH 6.0 (Tris-EDTA buffer, pH 9.0 was used to detect MMP-2). Endogenous peroxidase activity was blocked by incubating the samples in PBS containing $3 \% \mathrm{H}_{2} \mathrm{O}_{2}$, and the non-specific binding sites were blocked with $3 \%$ bovine albumin (Sigma-Aldrich) in PBS for $20 \mathrm{~min}$ at room temperature. The following primary monoclonal antibodies were added: MMP-2 (1:8000; Lab Vision Corp. Fremont, CA, USA), glial fibrillary acidic protein (GFAP) (1:4000, Lab Vision Corp), and p53 (1:8000; Santa Cruz Biotechnology). Then, a biotin-labeled secondary antibody (Dako, Carpinteria, CA, USA) was added, and the samples were incubated at room temperature for $1 \mathrm{~h}$. A streptavidin-horseradish peroxidase (Dako) detection system was applied to the capillary channels, followed by a $20 \mathrm{~min}$ incubation at room temperature. The tissue sections were ready for the chromogen reaction with diaminobenzidine. Counterstaining was performed with Harris hematoxylin. Control experiments were performed in all specimens at the time of immunostaining using the secondary antibody alone.

\section{In situ zymography}

In situ zymography was performed to localize gelatinolytic activity, essentially as described by Sbai et al. [21] and Miller et al. [22]. Eight-mm-thick sections were cut from the same tissue used for immunohistochemistry. The fixed, paraffin-embedded tissue sections were heated to $59{ }^{\circ} \mathrm{C}$ overnight, deparaffinized in xylene, and rehydrated in a graded alcohol series. The substrate was prepared by dissolving $1 \mathrm{mg}$ DQ gelatin in $1 \mathrm{ml}$ Milli-Q water, followed by a 1:50 dilution in a reaction buffer containing $50 \mathrm{mM}$ Tris- $\mathrm{HCl}, 150 \mathrm{mM} \mathrm{NaCl}, 5 \mathrm{mM} \mathrm{CaCl}_{2}$, and $0.2 \mathrm{mM}$ sodium azide ( $\mathrm{pH} 7.6)$. Of this mixture, $250 \mu \mathrm{l}$ was placed on top of the tissue sections, covered with Parafilm, and incubated in a dark humidity chamber at $37{ }^{\circ} \mathrm{C}$. The Parafilm was removed gently after $2 \mathrm{~h}$, and the sections were rinsed with Milli-Q water and fixed in $4 \%$ neutral-buffered formalin for $10 \mathrm{~min}$ in the dark.
Sample autofluorescence was quenched in a $100 \mathrm{mg} / \mathrm{ml}$ $\mathrm{NaBH}_{4}$ solution for $30 \mathrm{~min}$. The sections were rinsed in a PBS bath $(2 \times 5 \mathrm{~min})$ and mounted with glycerol containing DAPI to counterstain the nuclei. Control slides were pre-incubated with $20 \mathrm{mM}$ EDTA for $1 \mathrm{~h}$ to verify the contribution of MMPs. A 20 mM EDTA solution was also added to the substrate. Tissue autofluorescence level was evaluated by incubating control sections at $220{ }^{\circ} \mathrm{C}$ for $2 \mathrm{~h}$ immediately after the substrate had been added. Fluorescence was evaluated using an Olympus confocal laser microscope with Olympus Application Suite Advanced Fluorescence software (Tokyo, Japan).

\section{Statistical analyses}

Each experiment was repeated at least three times to confirm reproducibility. Comparisons were made using oneway analysis of variance. Differences between means were evaluated using the Kruskal-Wallis test, and p-values $<0.05$ were considered significant.

\section{Results \\ Effect of radiation on MMP-2 activity according to p53 status}

The human malignant glioma cell lines U87MG (wt p53) and U251 (mt p53) and the human osteosarcoma cell lines U2OS (wt p53) and SAOS (del p53) were selected to measure the effect of radiation on p53 expression and MMP-2 activity. As shown Fig. 1, MMP-2 activity and p53 expression increased in U87MG and $\mathrm{U} 2 \mathrm{OS}$ cells wt p53 in proportion to the radiation dose, but these were unchanged in U251 and SAO2 cells. TIMP-2 is an endogenous MMP-2 inhibitor, and the balance between TIMP-2 and MMP-2 is a critical determinant of malignant glioma invasion. We performed a Western blot for TIMP-2 in the four cell lines exposed to radiation. TIMP-2 expression did not change after radiation exposure in three of the cell lines, except U2OS. The sublethal radiation dose increased p53 expression and MMP-2 activity in U87MG cells without a corresponding increase in TIMP-2 $(p<0.05$, Fig. $1 \mathrm{a}, \mathrm{b}, \mathrm{c})$. In contrast, TIMP-2 expression and MMP-2 activity in U2OS cells with wt p53 increased simultaneously following radiation. These results suggest that TIMP-2 expression was likely to be different depending on the cell type, and that the imbalance between TIMP-2 and MMP-2 expression may enhanced tumor invasion. In addition, MMP-2 activity caused by radiation may be associated with p53 status.

\section{Radiation-induced p53 upregulation is responsible for promoting MMP2 activity}

MMP-2 activity and p53 expression simultaneously increased after radiation exposure, and the MMP-2 gene promoter region has a putative $\mathrm{p} 53$ binding site. Thus, 


\section{a. GM cell line}

U87MG (wt p53)

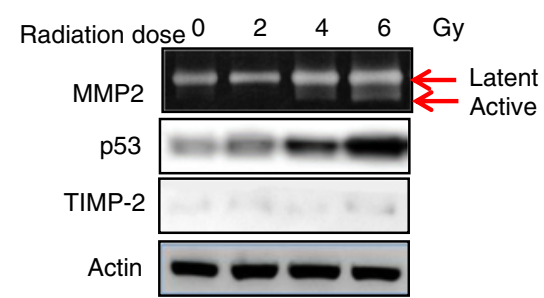

\section{U251 (mt p53)}

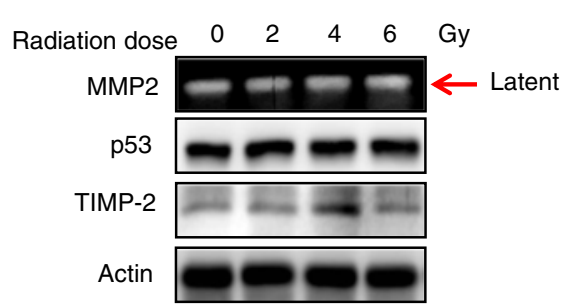

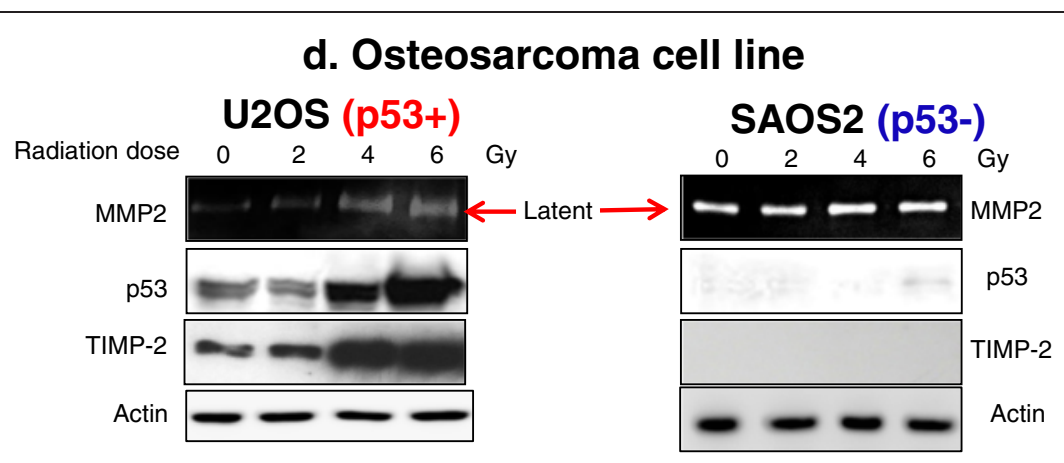

b. MMP-2 activity
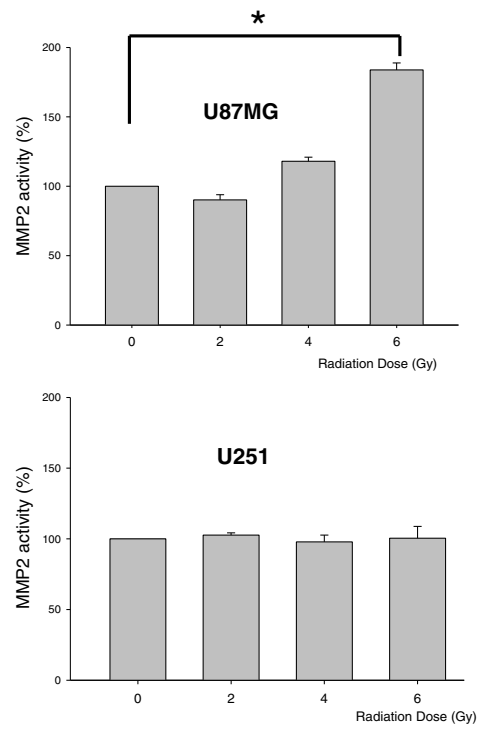

c. p53 expression
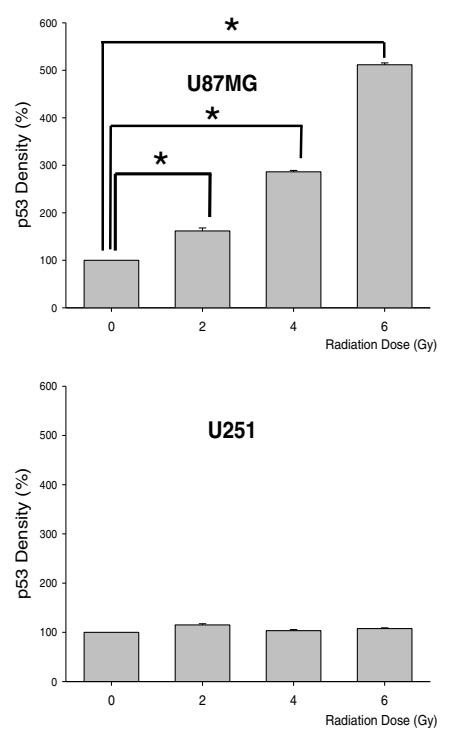

Fig. 1 Radiation effect on p53, TIMP2 expression and MMP-2 activity in 2 cell lines with wt p53 and 2 cell lines without p53. Each cell lines were exposed 0, 2, 4, 6 Gy, respectively. MMP-2 activity was detected using zymography (one lane) and expression of TIMP-2 and p53 was detected using western blot (2-4 lane) a Glioblastoma cell lines; b MMP-2 activity of U87MG and U251; c p53 density of U87MG and U251; d Osteosarcoma cell lines

we identified whether p53 actually enhanced radiationinduced MMP-2 activity. U87MG was transfected p53 siRNA oligonucleotide and was performed Western blot for p53 and TIMP-2 (Fig. 2a), and Zymography for determining of MMP-2 activity (Fig. 2b). p53 expression and MMP-2 activity increased in proportion to the radiation dose in control transfected scramble RNA to U87MG cells, but remained unchanged in p53 knockdown U87MG cells (Fig. 2a, b). TIMP-2 expression was not changed by radiation, and no difference in TIMP-2 expression was detected between the control and p53 knockdown U87MG cells.

Establishment of a bioluminescent tumor cell line We established the bioluminescent tumor cell line "U87-Fluc" for in vivo experiments, in which u87MG was transfected with firefly luciferase (Fluc). This is a useful tool to observe tumor formation without sacrifice after implanting tumor cells in the mouse brain. The U87-Fluc cell line emitted photons when Dluciferin was added (Fig. 3a), and U87-Fluc cells increased p53 expression and MMP-2 activity in proportion to the radiation dose but did not affect TIMP2 expression (Fig. 3b, c). MMP-2 activity increased depending on the incubation time after radiation exposure and showed a great difference between control and radiated cells after $24 \mathrm{hr}(p<0.05$, Fig. $3 \mathrm{~d})$.

Tumor growth rate analyses using optical bioluminescence imaging

U87-Fluc-bearing nude mice brains were observed continuously by tumor bioluminescence using the IVIS 100 imaging system to determine the effects of radiation on tumor growth. Additional file 1: Figure S1 illustrates the 


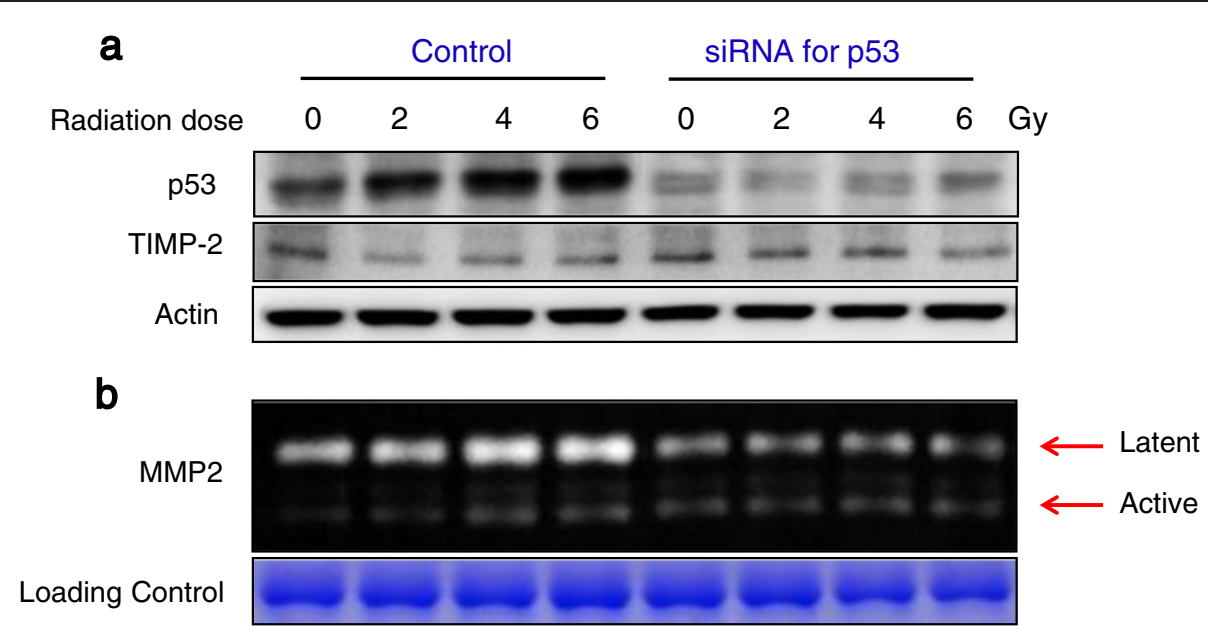

Fig. 2 Radiation effect on p53, TIMP2 expression and MMP-2 activity in p53 knockdown U87MG. 'Control' Scramble RNA transfected U87MG, 'siRNA for p53' is p53 knock-downed U87MG using siRNA technology. a Western blot; b Zymography for MMP-2 activity

process used to assess the radiation effects on brain tumors in vivo. The mouse brains were exposed to radiation after tumor formation had been observed by tumor bioluminescence. As shown in Fig. 4, notable retardation of tumor growth was observed in the single 6 Gy exposed group, compared with that in the control groups. However, the brain tumors continued to grow, and the final growth rate was similar to that of the control group from about day 20. No difference was found in the radiation-fractionated group, compared to the control group, indicating that the single dose (6 Gy) treatment or low dose fractionated irradiation was insufficient for complete control of tumor growth.

\section{Irradiation promotes glioma cell dissemination in vivo}

The observed invasive effect of sublethal irradiation in U87-Fluc cells in vivo may have clinical relevance. As shown in Fig. 5 tumors in the non-radiated group had clear borders, whereas in the radiated group showed more irregular margin, the diffuse infiltration of tumor cells into surrounding normal brain and exhibited multiple tumor satellites. a

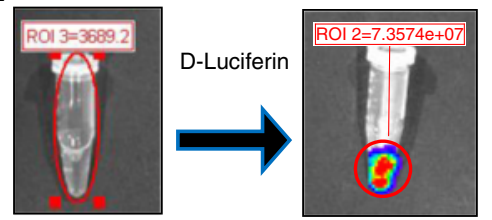

b

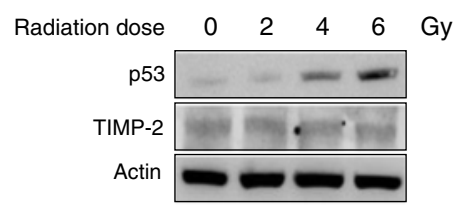

C

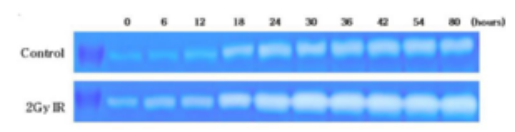

d

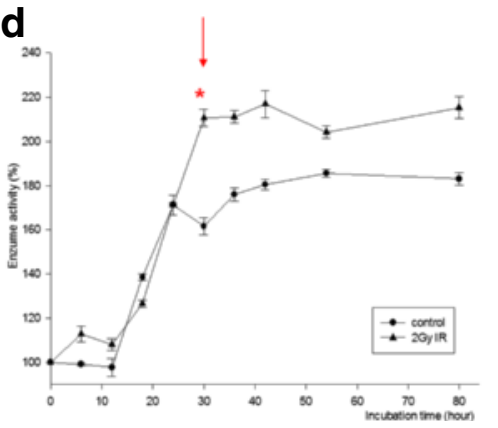

Fig. 3 Radiation effect on p53, MMP-2 and TIMP-2 in U87 MG-Fluc cells. Establishment bioluminescent tumor cells "U87-Fluc" which is transfected with firefly luciferase (fluc) in u87MG. a Photons emitted from U87-Fluc cell line when D-luciferin was added; b Western blot; c Zymography for detection MMP-2 activity in dependent on time; $\mathbf{d}$ Density of MMP-2 activity. It had been showed statistical significance between control and 2 Gy IR from 30 hr $(p<0.05)$ 

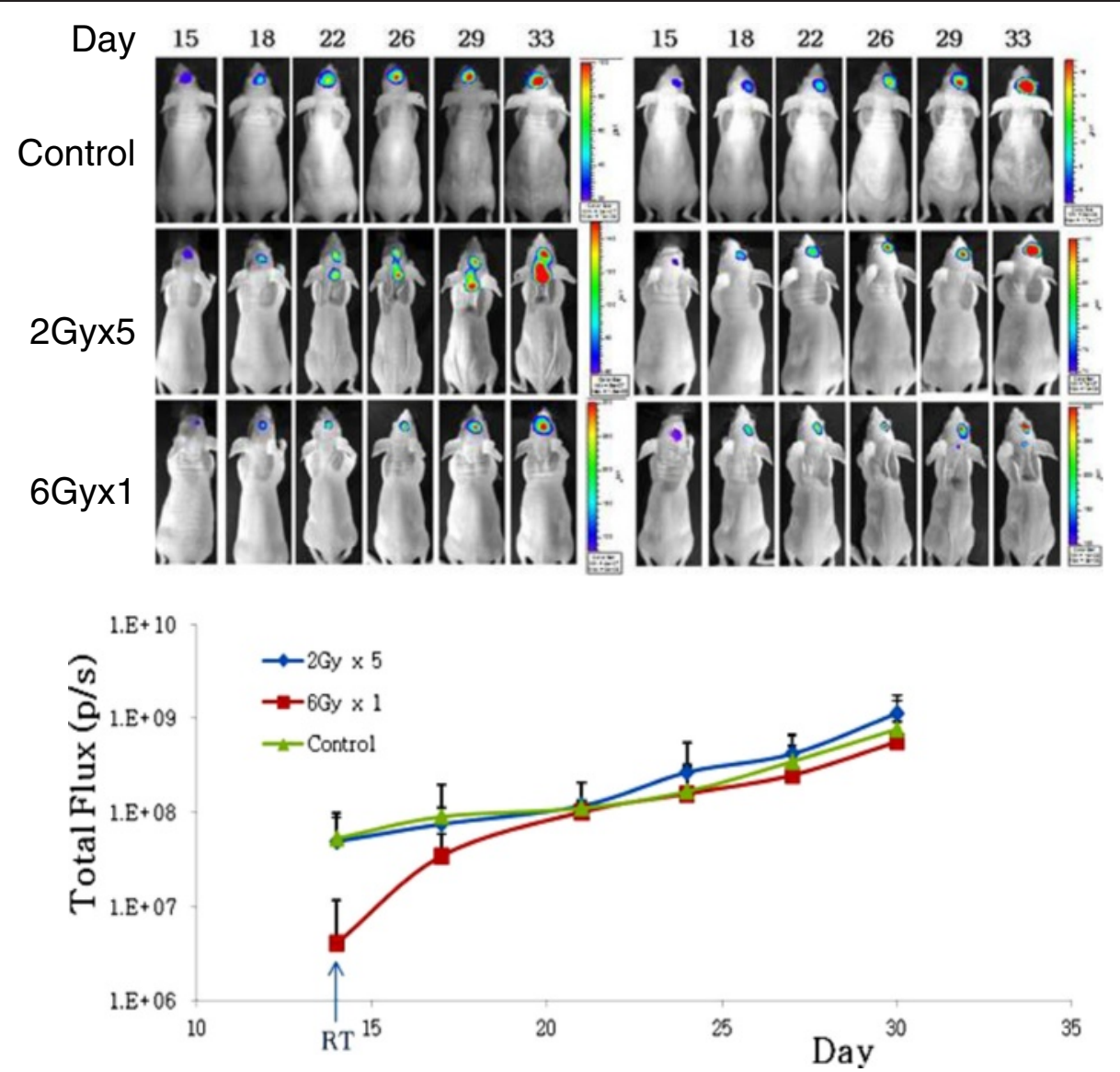

Fig. 4 Tumor growth rate analyses using Optical Bioluminescence Imaging. U87-Fluc bearing nude mice brain were continuously observed through tumor bioluminescence by the IVIS 100 imaging system. For pilot study we designated control group (non-radiated group) and two groups (radiated group). Among radiated group, one group was exposed to fractionated dose radiation (2Gy $\times 5$ ) and another group was exposed to single dose radiation (6 Gy)

The in vitro results showed an association between p53 and MMP-2. We wanted to confirm whether tumor cells invading after radiation exposure was related with p53 expression and MMP-2 activity. Thus, we verified that the invasive cells surrounding normal brain were malignant glioma cells using GFAP staining as a glial cell marker, and performed immunohistochemistry for p53 and MMP-2. p53 was highly expressed in irradiated tumors and was particularly distributed in invasive tumor cells around the margin area (Fig. 6), even though no difference was observed between the single and the fractionated dose groups. GFAP and p53-positive cells were distributed similarly.

In situ zymography was performed in radiationexposed and unexposed nude mouse brains. This is a unique technique that enables localization of gelatinolytic activities in histological sections. Notably, autofluorescence during in situ zymography was minimized by $\mathrm{NaBH} 4$. Green fluorescence as a signal for gelatinolytic activity was found around the tumor margin area in the radiated group but not in non-radiated group (Fig. 7c, d).
However, MMP-2 expression showed no difference between the two groups (Fig. 7b).

\section{Discussion}

Primary GBM represents about $90 \%$ of GBMs, and the wild-type p53 gene is present in about $70 \%$ of primary GBMs. However, p53 occurs less frequently in secondary GBMs (35\%) [23]. p53 mutations frequently occur in low grade gliomas (WHO grade II astrocytoma) [24] and, thus, are a frequent event in the pathological progression of secondary GBM (WHO Grade IV) [25]. Secondary GBM arises from a preexisting grade II or III astrocytoma, whereas a primary GBM forms de novo. p53 mutations were detected in only $40 \%$ of the samples, predominantly in anaplastic astrocytomas, indicating that $>50 \%$ of patients have wild type p53 [21].

In this study, we identified RT effect on migration and invasion of malignant glioma and studied the association between p53 and MMP-2 through in vitro and in vivo study. p53 is well known as a radio-sensitizer and radiation induces p53 expression. And it has been 


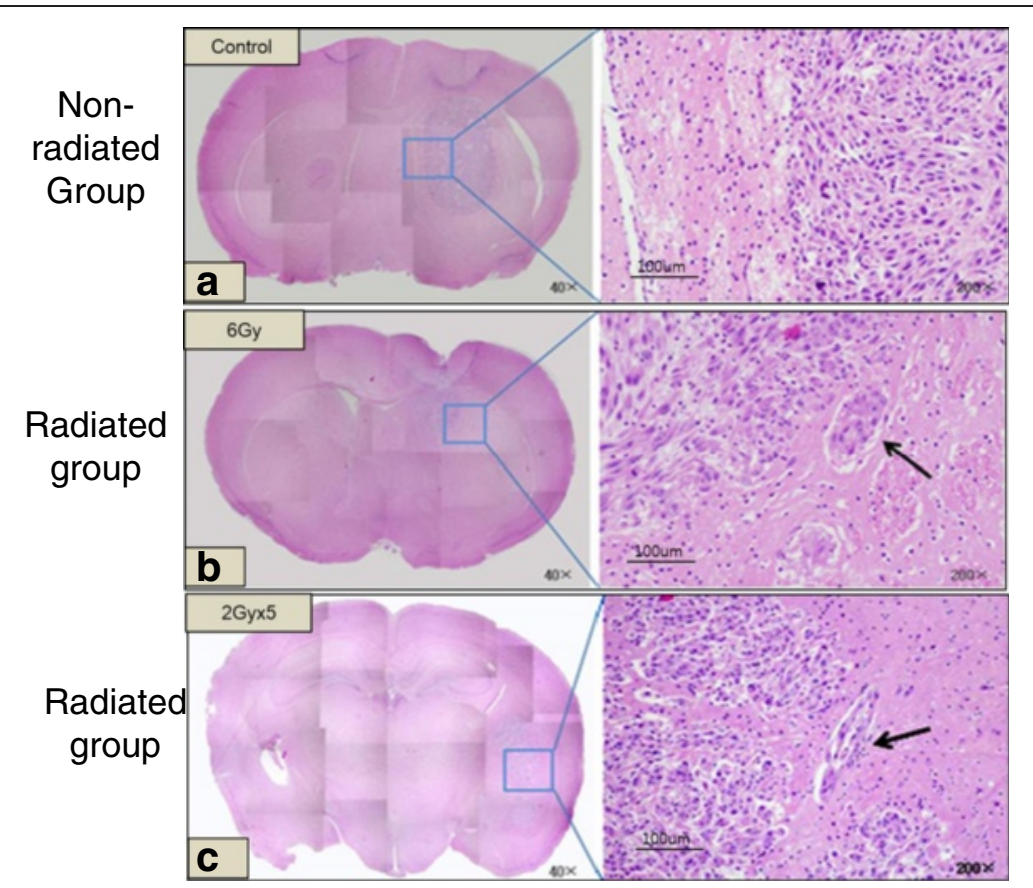

Fig. 5 Hematoxyin and Eosin staining of brain tissues. Exposure to radiation was more invasive and exhibited multiple tumor cell satellites, while non-radiated tumor showed less invasiveness and clear margin. a Non-radiated group, b Radiated group (Single dose), c Radiated group (fractionation)

hypothesized that RT may promote tumor metastasis in a subset of human cancers harboring the wild-type p53 gene [3]. Increased p53 expression affects the metastatic potential of certain types of cancers $[15,22]$. Radiation-induced p53 upregulation is responsible for promoting the VEGF-MMP2 pathway involved in enhancing the invasiveness of both irradiated and bystander hepatoma cells [26].

MMPs are a family of extracellular matrix (ECM)-degrading enzymes associated with numerous physiological and pathological events, such as malignant tumor cell invasion processes $[8,9]$. And MMP activity is regulated at multiple levels, such as at the level of gene transcription and the synthesis of pro-MMPs. Ionizing radiation is known as a representative of DNA-damaging agents. The DNA cleavage by irradiation leads to the accumulation of p53 and its translocation into the nucleus [27, 28].

Then, the activated p53 binds to DNA in a sequencespecific manner and modulates a set of genes [29]. Other study has demonstrated that the promoter region of the MMP-2 gene has a putative p53 binding site and that wild-type p53 transcriptionally upregulates the expression of MMP-2 mRNA [14, 22]. Considering the above references, we examined the radiation effect on MMP-2 activity and association between p53 status and MMP-2 activity. In our study, MMP-2 activity was actually increased depending on the radiation dose in cell lines with wt p53 gene; however, no change of MMP-2 activity was shown in cell lines with mt p53 gene. So we expected that p53 increased by radiation could upregulate MMP-2 activities, then confirmed their association using p53 knock-downed u87MG cells by siRNA technology. MMP-2 activity was decreased regardless of the radiation dose in p53 knockdown, while increased depending on the radiation dose in the control. Even though further studies are required to identify direct association between p53 and MMP-2 activity, p53, at least, can modulate the change of MMP-2 activity by radiation on the basis of the present study.

Many studies have reported the effects of radiation on tumor cell migration, invasion, and surrounding healthy tissue. Radiation enhances cell invasion and induces MMP-2 and -9 activities in various tumors and in lung epithelial cells [30-32]. Surrounding the brain tissue exposed to radiation secretes several mediators, such as VEGF, MMPs, cyclooxygenase-2, and transforming growth factor-beta, all of which play vital roles in tumor invasion and metastasis [26, 33, 34]. An imbalance between MMP2 and TIMP-2 has an important role in glioma invasion by degradation of the ECM [34, 35]. TIMP-2 is an endogenous MMP-2 inhibitor and critical for maintenance of tissue homeostasis by suppressing proliferation of quiescent cells in response to angiogenic factors, and by inhibiting protease activities in tissues undergoing ECM remodeling. Our results showed that the expression of TIMP-2 was not changed, while MMP-2 activity increased depending on 


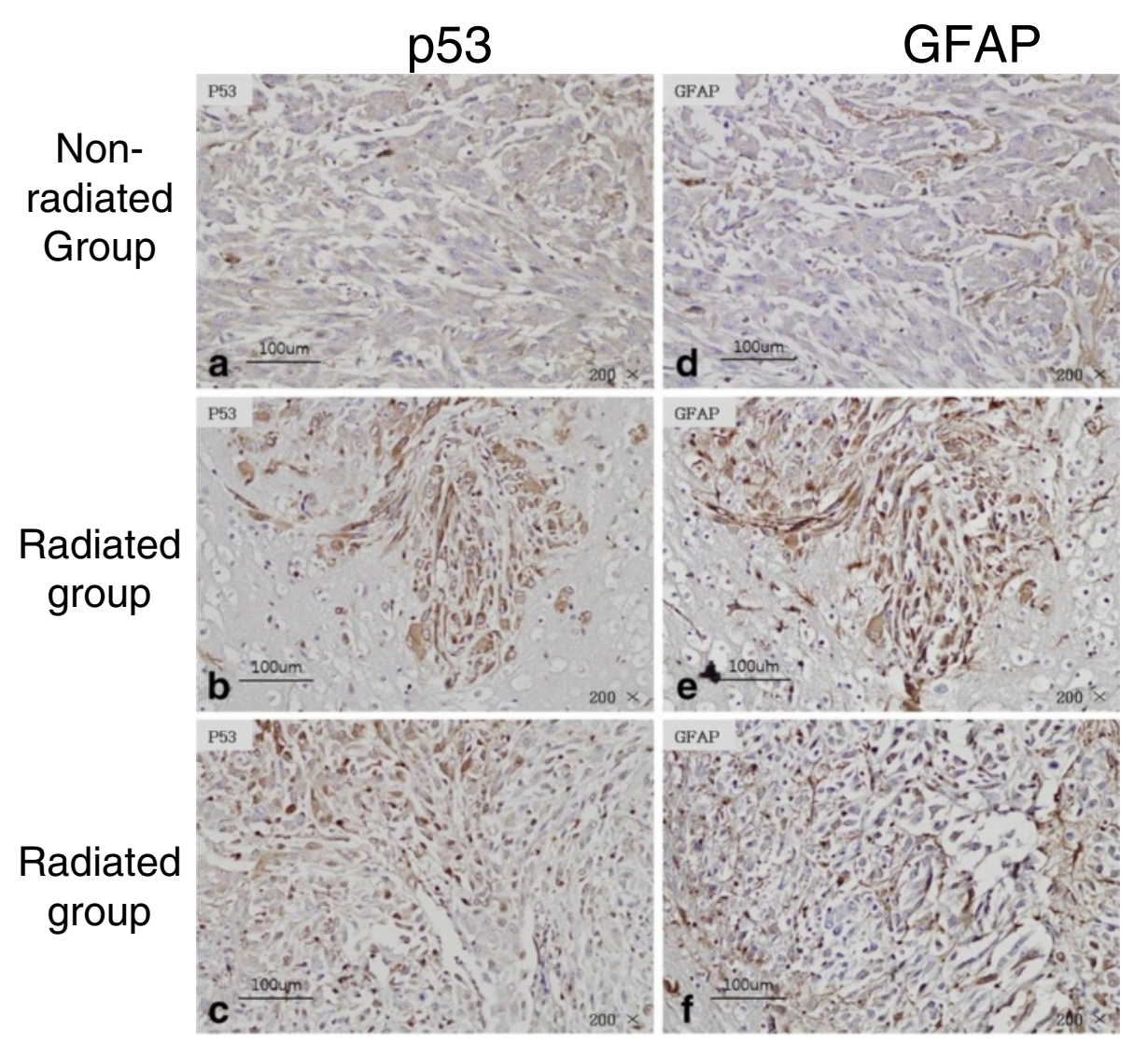

Fig. 6 Immunohistochemistry for p53 and GFAP in brain tissues. GFAP (d,e,f) as a glial cell marker was stained to confirm whether p53 (a,b,c) positive cells were glioma cell or not

the radiation dose in malignant glioma cell lines. We also obtained the same results through in vivo experiments. The radiation-exposed U87-Fluc-bearing mouse brain was significantly more invasive, while MMP-2 and p53 expression, as well as gelatinolytic activity, increased, particularly in margin areas. However, TIMP-2 expression increased along with MMP-2 activity in U2OS cells. Those results suggest that TIMP-2 expression was likely to be different depending on the organ specific cell type, and that the imbalance between TIMP-2 and MMP-2 in malignant glioma with wt p53 may be important to understanding radiationenhanced glioma invasion. Finally, we confirmed that radiation could induce glioma invasion through in vivo brain tumor model using U87-Fluc cell with bioluminescent. The U87-Fluc-bearing mouse brain was observed continuously using tumor bioluminescence with the IVIS $100 \mathrm{im}$ aging system. This is a useful tool to observe tumor formation without sacrificing animals after implanting tumor cells. As shown through the results, radiation significantly enhanced tumor cell invasion in the U87-Flucbearing mouse brain tumor model. We suggest that invasive brain tumor model caused by RT could be applied to in vivo studies for verification of the effect of anti-invasive therapeutic target genes or drugs.

The clinical implications of this study are as follows:

a) Alterations of radiotherapy for human malignant glioma might be considered to determine whether the patient is harboring wild-type p53 or mutant type.

b) We propose using an MMP inhibitor adjunctively to suppress the radiation-induced increase in MMP-2 expression and to prevent unexpected or unwanted tumor invasion due to RT.

\section{Conclusions}

Radiation enhanced glioma invasion in the mouse brain tumor model. Wild type p53 induced by raidation modulated MMP-2 activity, and the imbalance between MMP-2 and TIMP-2 may have an important role in glioma invasion by degradation of the ECM. For the study using mouse brain tumor model, bioluminescent U87-Fluc was useful to observe tumor formation without sacrificing animals after implanting tumor cells. Taken together, these 


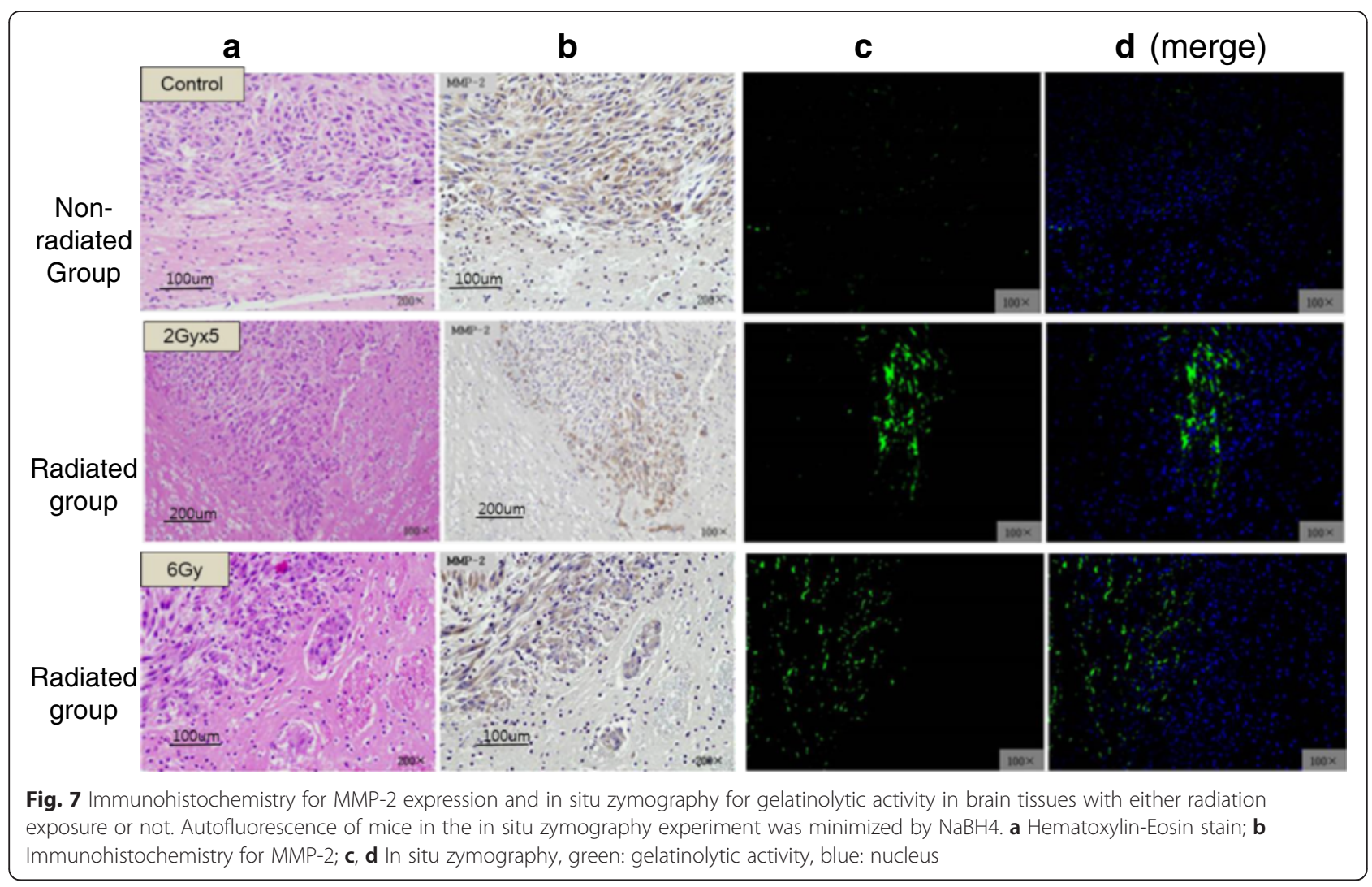

findings suggest that involved-field RT for malignant gliomas may need to be reconsidered. Future trials should consider pharmacological therapies that inhibit invasion concurrently with RT.

\section{Additional file}

Additional file 1: Figure S1. The illustration of the experimental process to assess radiation effect on brain tumor in vivo. After implantation U87-Fluc cells into brain, tumor bioluminescence of mouse brain bearing U87-Fluc was continuously observed from day 10 to 28 using the IVIS 100 imaging system. Radiation was exposed to either Single dose 6Gy or 2Gy fractions 5 times for 7 days on 14 day after U87-Fluc implantation. Mice brain were performed with Hematoxylin and eosin or immunohistochemical staining after execution on 30 day. (PPTX $381 \mathrm{~kb}$ )

\section{Abbreviation}

GBM: Glioblastoma; RT: RadioTherapy; wt: Wild type; mt: Mutant type; del: deletion; MMP: Matrix metalloproteinase; TIMP: Tissue inhibitor of Matrix metalloproteinase; EDTA: Ethylenediaminetetraacetic acid.

\section{Completing interests}

The authors declare that they have no competing interests.

\section{Author's contribution}

JS, PJ, JWW and PYH designed and planned the experiments. PJ, RHH, YSW and $\mathrm{YCH}$ performed the experiments. JS, PJ, WM and $\mathrm{RHH}$ evaluated the data and wrote the manuscript. All co-authors approved the manuscript. JTY, MKS and KIY provided technical help and writing assistance.

\section{Acknowledgements}

This study was supported by the Leading Foreign Research Institute Recruitment Program through the National Research Foundation of Korea (NRF), funded by the Ministry of Education, Science, and Technology (MEST) (2011-0030034)

Received: 6 February 2015 Accepted: 26 July 2015

Published online: 06 August 2015

\section{References}

1. Vehlow A, Cordes N. Invasion as target for therapy of glioblastoma multiforme. Biochim Biophys Acta. 2013;1836(2):236-44.

2. Badiga AV et al. MMP-2 siRNA inhibits radiation-enhanced invasiveness in glioma cells. PLoS One. 2011;6(6):e20614.

3. Garden AS et al. Outcome and patterns of failure following limited-volume irradiation for malignant astrocytomas. Radiother Oncol. 1991;20(2):99-110.

4. Hess CF et al. Malignant glioma: patterns of failure following individually tailored limited volume irradiation. Radiother Oncol. 1994;30(2):146-9.

5. Kil WJ, Tofilon PJ, Camphausen K. Post-radiation increase in VEGF enhances glioma cell motility in vitro. Radiat Oncol. 2012;7:25

6. Yano $S$ et al. Invading cancer cells are predominantly in $\mathrm{G} / \mathrm{G} 1$ resulting in chemoresistance demonstrated by real-time FUCCI imaging. Cell Cycle. 2014;13(6):953-60

7. Zhou W et al. Attention to normal brain volumes in glioblastoma radiotherapy: potential role in tumor invasion and vasculogenesis. Med Hypotheses. 2013;80(4):501-4.

8. Sternlicht MD, Werb Z. How matrix metalloproteinases regulate cell behavior Annu Rev Cell Dev Biol. 2001;17:463-516.

9. Egeblad M, Werb Z. New functions for the matrix metalloproteinases in cancer progression. Nat Rev Cancer. 2002;2(3):161-74.

10. Wild-Bode $C$ et al. Sublethal irradiation promotes migration and invasiveness of glioma cells: implications for radiotherapy of human glioblastoma. Cancer Res. 2001;61(6):2744-50. 
11. Nirmala C et al. Effects of radiation on the levels of MMP-2, MMP-9 and TIMP-1 during morphogenic glial-endothelial cell interactions. Int J Cancer. 2000;88(5):766-71.

12. Araya J et al. lonizing radiation enhances matrix metalloproteinase-2 production in human lung epithelial cells. Am J Physiol Lung Cell Mol Physiol. 2001:280(1):L30-8.

13. Grelewski PG, Bar JK. The role of p53 protein and MMP-2 tumor/stromal cells expression on progressive growth of ovarian neoplasms. Cancer Investig. 2013;31(7):472-9.

14. Bian J, Sun Y. Transcriptional activation by p53 of the human type IV collagenase (gelatinase A or matrix metalloproteinase 2) promoter. Mol Cell Biol. 1997;17(11):6330-8.

15. Cheng $L$ et al. $p 53$ alteration in regional lymph node metastases from prostate carcinoma: a marker for progression? Cancer. 1999:85(11):2455-9.

16. Lee $\mathrm{YJ}$ et al. Enhanced radiosensitization of p53 mutant cells by oleamide. Int J Radiat Oncol Biol Phys. 2006;64(5):1466-74.

17. Levine AJ, Momand J, Finlay CA. The p53 tumour suppressor gene. Nature. 1991;351(6326):453-6.

18. Lowe SW. Cancer therapy and p53. Curr Opin Oncol. 1995;7(6):547-53.

19. Okaichi $\mathrm{K}$ et al. A point mutation of human $\mathrm{p} 53$, which was not detected as a mutation by a yeast functional assay, led to apoptosis but not p21Waf1/ Cip1/Sdi1 expression in response to ionizing radiation in a human osteosarcoma cell line, Saos-2. Int J Radiat Oncol Biol Phys. 1999:45(4):975-80.

20. Ra HJ, Parks WC. Control of matrix metalloproteinase catalytic activity. Matrix Biol. 2007;26(8):587-96

21. Milinkovic $V$ et al. Identification of novel genetic alterations in samples of malignant glioma patients. PLoS One. 2013;8(12):e82108.

22. Muller PA, Vousden KH, Norman JC. p53 and its mutants in tumor cell migration and invasion. J Cell Biol. 2011;192(2):209-18.

23. Ohgaki $\mathrm{H}$ et al. Genetic pathways to glioblastoma: a population-based study. Cancer Res. 2004;64(19):6892-9.

24. Figarella-Branger $D$ et al. Histomolecular classification of adult diffuse gliomas: the diagnostic value of immunohistochemical markers. Rev Neurol (Paris). 2011;167(10):683-90.

25. Gladson CL, Prayson RA, Liu WM. The pathobiology of glioma tumors. Annu Rev Pathol. 2010:5:33-50

26. He M et al. Radiation enhances the invasiveness of irradiated and nonirradiated bystander hepatoma cells through a VEGF-MMP2 pathway initiated by p53. Radiat Res. 2013;180(4):389-97.

27. Shieh SY et al. DNA damage-induced phosphorylation of p53 alleviates inhibition by MDM2. Cell. 1997;91(3):325-34.

28. Canman CE, Lim DS. The role of ATM in DNA damage responses and cancer. Oncogene. 1998:17(25):3301-8.

29. Agarwal ML et al. The p53 network. J Biol Chem. 1998;273(1):1-4.

30. Sbai $O$ et al. Vesicular trafficking and secretion of matrix metalloproteinases$2,-9$ and tissue inhibitor of metalloproteinases-1 in neuronal cells. Mol Cell Neurosci. 2008:39(4):549-68.

31. Miller DW et al. Rapid vessel regression, protease inhibition, and stromal normalization upon short-term vascular endothelial growth factor receptor 2 inhibition in skin carcinoma heterotransplants. Am J Pathol. 2005;167(5):1389-403.

32. Zaboronok $\mathrm{A}$ et al. Proton beam irradiation stimulates migration and invasion of human U87 malignant glioma cells. J Radiat Res. 2014;55(2):283-7

33. Shiao SL, Coussens LM. The tumor-immune microenvironment and response to radiation therapy. J Mammary Gland Biol Neoplasia. 2010;15(4):411-21

34. Zheng $\mathrm{H}$ et al. p53 and Pten control neural and glioma stem/progenitor cell renewal and differentiation. Nature. 2008;455(7216):1129-33.

35. Lee WH et al. Irradiation alters MMP-2/TIMP-2 system and collagen type IV degradation in brain. Int J Radiat Oncol Biol Phys. 2012;82(5):1559-66.

\section{Submit your next manuscript to BioMed Central and take full advantage of:}

- Convenient online submission

- Thorough peer review

- No space constraints or color figure charges

- Immediate publication on acceptance

- Inclusion in PubMed, CAS, Scopus and Google Scholar

- Research which is freely available for redistribution

Submit your manuscript at www.biomedcentral.com/submit 